\title{
The study of the dependence of mechanical properties and fracture of water-saturated high- strength concrete on the parameters of pore structure
}

\author{
Igor Konovalenko ${ }^{1,2, *}$, Evgeny Shilko ${ }^{2,3}$, and Ivan Konovalenko ${ }^{1,4}$ \\ ${ }^{1}$ National Research Tomsk Polytechnic University, 634050 Tomsk, Russian Federation \\ ${ }^{2}$ Institute of Strength Physics and Materials Science SB RAS, 634055 Tomsk, Russian Federation \\ ${ }^{3}$ National Research Tomsk State University, 634050 Tomsk, Russian Federation \\ ${ }^{4}$ Tomsk Agricultural Institute - a branch of FSBEI HE Novosibirsk SAU, 634050 Tomsk, Russian \\ Federation
}

\begin{abstract}
Based on the hybrid numerical method, namely, the hybrid method of movable cellular automata, we developed a computer-based two-scale mechanical model of heavy water-saturated concrete. The model takes into account the content of the liquid phase and its redistribution in networks of microscopic and capillary pores. The features of deformation and fracture of concrete samples under uniaxial compression were numerically studied in a wide range of variation of the macroscopic characteristics of the pore space, viscosity of an interstitial liquid and loading rate. Simulation results confirmed the possibility of a unified description of the dependence of the strength of heavy concrete on the sample permeability and size, fluid viscosity and the loading rate in terms of the complex dimensionless parameter that combines these characteristics.
\end{abstract}

\section{Introduction}

Currently, a significant part of building structures that work in contact with water, including platform supports and various port facilities, are made of high-strength concrete. Under conditions of being in the water, the two-scale pore space of concrete (microscopic and capillary pores) is usually filled with water. Pore water (interstitial liquid) is considered to be responsible for the numerous phenomena of premature destruction of concrete elements under loads that are significantly smaller than the critical values for dry concrete structures. The effect of interstitial liquid on the mechanical characteristics of concrete (in particular, a strength reduction) is characterized by two aspects: the physicochemical, known as adsorption decrease in strength, and the mechanical, related to action of pore pressure on the stress state of a solid-phase skeleton. Note that the effect of the strength reduction takes place not only for concrete, which stays in contact with water for a long time and undergoes

\footnotetext{
*Corresponding author: iskonovalenko@tpu.ru
} 
its destructive physicochemical effect but also for samples and structural elements in water for a limited time, insufficient for the chemical degradation of the solid-phase skeleton.

Based on the above, it is important to investigate the mechanical effect of pore water on the stress state and strength of water-saturated concrete and, in particular, to analyze the interrelation of parameters of its pore structure and characteristics of mechanical behavior. Such studies are particularly relevant for predicting the mechanical characteristics of concrete under conditions of dynamic loading when the characteristic times of change in the stress state of the skeleton are comparable to the characteristic times of fluid redistribution in the pore space of concrete. It is extremely difficult to experimentally determine the mechanical contribution of fluid in the pore space of each of the scales (microscopic and capillary) to the macroscopic stress-strain state of concrete. Therefore, in the present paper, this problem was solved by computer simulation.

Currently, a computer study of the mechanical properties and fracture of elastic and elastoplastic heterogeneous materials under mechanical loading is successfully carried out based on two different approaches: discrete [1-3] and continuum [4-6]. The study of porous fluid-saturated materials based on classical models of heterogeneous solids is very difficult, in particular, due to the need to simultaneously solve several problems: deformation of the porous skeleton, fluid filtration in the pore space and the mutual influence of these processes. In this connection, hybrid technique, namely the hybrid method of movable cellular automata, was used as an effective method of modeling, which allows solving these problems and directly studying the processes of localized and multiple accumulations of damages and crack growth. This method combines the advantages of the particle method (in the formulation of discrete elements) and the grid method and makes it possible to simulate the behavior of permeable fluid-saturated materials with a multiscale pore structure [7].

\section{Model description}

The object of the study was heavy concrete. In the works [8] it is shown that under a theoretical study of the mechanical behavior of concrete at the mesoscale, the number of its components in the model can be reduced. In particular, aggregates with a characteristic size of less than $5 \mathrm{~mm}$ (small inclusions) may not be explicitly taken into account in the model. Accordingly, the cement stone (binder containing such small aggregates) is assumed to be a homogeneous isotropic medium with some effective mechanical characteristics. Larger aggregates are taken into account explicitly. Such a representation of concrete as a twocomponent material is a common mesoscopic model $[9,10]$. Thus, in the developed model of concrete, it was assumed that its main components are large inclusions (crushed basalt), cement stone and interstitial liquid.

In the framework of the hybrid movable cellular automaton method, a two-scale mechanical coupled model of a liquid-saturated heavy concrete was developed. It takes into account the presence of brittle high-strength reinforcing inclusions (basalt aggregates), a pore network of two different scales (communicating micropores of characteristic sizes hundreds of micrometers and capillary pores of characteristic sizes less than a micrometer) in the cement stone, as well as the possibility of redistribution of interstitial liquid in the pore networks under mechanical loading of concrete. Micropores were taken into account explicitly (as rounded voids in the cement stone), while capillary porosity was taken into account implicitly by specifying integral parameters (capillary porosity and permeability).

The mechanical interaction of the solid-phase skeleton of concrete and pore liquid was taken into account based on the Biot's poroelasticity model [11,12] using the effective Terzaghi stresses $\sigma_{\alpha \beta}^{e f f}=\sigma_{\alpha \beta}+\delta_{\alpha \beta} \lambda P$. Here, $\sigma_{\alpha \beta}$ is the component of the local stress tensor 
in the solid-phase skeleton $(\alpha, \beta=\mathrm{x}, \mathrm{y}, \mathrm{z}), \delta_{\alpha \beta}$ is Kronecker delta, $P$ is local pore pressure, $\lambda$ is dimensionless coefficient of the mechanical influence of interstitial liquid. Model inclusions of basalt were assumed to be elastic-brittle, and cement stone was assumed to be elastic-plastic. Their mechanical behavior was described with the generalized Hooke's law for isotropic materials and the Nikolaevsky plasticity model [13,14]. Note that the Nikolaevsky model is a well-known dilatant model of the plasticity of brittle materials whose macroscopic inelastic behavior is associated with the nucleation and development of a network of local discontinuities. The fracture of basalt and cement stone was described on the basis of the Drucker-Prager criterion, which takes into account the influence of the local value of the average stress on the local shear strength $[15,16]$. For the interfaces of the inclusions of basalt and cement stone, the Drucker-Prager strength criterion was also used with coefficients corresponding to the fracture criterion for cement stone. Note that for the porous component of concrete (cement stone), the criteria for strength and plasticity are formulated using the effective mean stress $\sigma_{\text {mean }}^{\text {eff }}=\sigma_{\text {mean }}+\lambda P_{\text {pore }}$ instead of the "classical" mean stress $\sigma_{\text {mean }}$ determined by external load on the local volume. The pore liquid was linearly compressible. The bulk compression module corresponded to fresh water $\left(K_{\text {fluid }}=2.2 \mathrm{GPa}\right)$. The gradient of pore pressure was considered as the driving force of fluid filtration in the pore space. Filtration was mathematically described on the basis of the classical equation of transport of fluid density [17]. Implementation details of the coupled model within the hybrid cellular automaton method are provided in the paper [7].

The problem was solved in a two-dimensional formulation under plain-strain condition. A series of plain square samples $(10 \mathrm{~cm} \times 10 \mathrm{~cm})$ of fluid-saturated concrete with only capillary porosity (recall that it is parametrically set in the model) were generated. Uniaxial compression was simulated with lubrication of the end faces of the sample and punches. The pore channels on the side surfaces of the sample were open, and liquid could flow freely from the sample into the surrounding space.

All model samples were characterized by the following constant values of the main structural and mechanical parameters: the average size of the inclusions of basalt is 10-15 $\mathrm{mm}$, the volume content of basalt is $10 \%$, and the capillary porosity $(\gamma)$ is $6 \%$. The initial water content in the cement stone is $17.14 \mathrm{~g} / \mathrm{kg}$. This correspond to the initial pore pressure equal to atmospheric $(P=0.1 \mathrm{MPa})$. Young's modulus $(E)$ and Poisson's ratio $(v)$ were: $88 \mathrm{GPa}$ and 0.23 for basalt, $40 \mathrm{GPa}$ and 0.18 for cement stone. The parameters of the Drucker-Prager strength criterion (compressive strength $\sigma_{c}$ and tensile strength $\sigma_{t}$ ) were specified as follows: $120 \mathrm{MPa}$ and $30 \mathrm{MPa}$ for cement stone, $260 \mathrm{MPa}$ and $52 \mathrm{MPa}$ for basalt. In the study, the values of the following model parameters were varied: the characteristic diameter of the capillary pore channels in the cement stone $\left(d_{\mathrm{ch}}\right)$ from $0.1 \mu \mathrm{m}$ to $1 \mu \mathrm{m}$, capillary permeability $\left(k=\gamma \times d_{\mathrm{ch}^{2}}\right)$ from $6 \times 10^{-16} \mathrm{~m}^{2}$ to $6 \times 10^{-14} \mathrm{~m}^{2}$, sample deformation rate $(\dot{\varepsilon})$ from $10^{-2}$ to 5 , fluid viscosity $(\eta)$ from $2 \times 10^{-4} \mathrm{~Pa} \cdot \mathrm{s}$ to $3.4 \times 10^{-3} \mathrm{~Pa} \cdot \mathrm{s}$.

\section{Simulation results}

The simulation results showed that the strength of mesoscale samples of water-saturated concrete is a single-value function of the ratio of two strain rates: the applied strain rate (the rate of deformation of the sample) and the characteristic rate of fluid filtration in the pore space. The dimensionless parameter characterizing this ratio is the analogue of the Darcy number [18]

$$
D a=\frac{\eta \dot{\varepsilon} W^{2}}{k \Delta P}
$$


where $W$ is the characteristic filtration distance (sample half-width), $\Delta P$ is the characteristic value of the difference in pore pressure at the distance $W$ (between the sample center and the lateral surface). Since during the deformation of the sample, the value of $P$ is continuously changing, the parameter $\Delta P$ in (1) was considered as some normalization constant. In the results presented below, its value was set equal to the initial pore pressure of the interstitial liquid $(\triangle P=0.1 \mathrm{MPa})$.

A typical form of the dependence of the uniaxial compressive strength of the sample $\sigma_{U C T}$ on the analogue of the Darcy number is obtained. It has a logistic profile and is approximated with good accuracy by a sigmoidal function with an exponent close to 2 . Figure 1 shows a typical $\sigma_{U C T}(D a)$ dependence for a sample containing only capillary pores (the volume content of micropores is zero). This dependence is characterized by the presence of three characteristic sections. The first section $(D a<600)$ corresponds to such a combination of permeability, liquid viscosity and loading rate, at which the liquid has time to be redistributed in the pore volume of the sample and flow out of the sample, i.e. to keep pore pressure at a low level. In this case, the pore fluid has almost no mechanical effect on the skeleton, and the strength of the sample is close to the maximum value and varies slightly. The third section $(D a>10000)$ corresponds to such a combination of the values of these parameters, at which the liquid does not have time to be redistributed in the capillary network during the loading of concrete sample. In this case, the porosity is actually closed, and the fluid creates excessive pore pressure, reducing the strength of the concrete as much as possible. Therefore, in the interval $D a>10000$, the strength of concrete is close to the minimum value (corresponding to a saturated sample with a system of isolated pores [7]) and varies very little. The second section of this dependence $(600<D a<10000)$ is a transitional one.

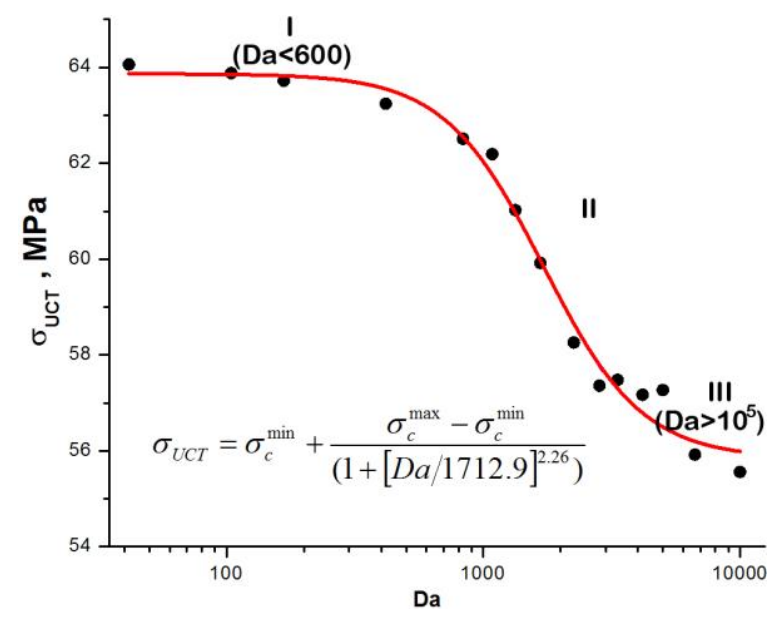

Fig. 1. Numerically derived dependence of compressive strength $\sigma_{U C T}$ of model samples of concrete with only capillary pores on the analogue of the Darcy number $D a$. Points are numerically obtained strength values. The solid curve is the approximating logistic curve with the following parameters: $\sigma_{\mathrm{c}}{ }^{\mathrm{min}}=55,8 \mathrm{MPa}$ (the minimum strength value of a concrete sample, achieved under condition of negligible rate of filteration), $\sigma_{\mathrm{c}}{ }^{\mathrm{max}}=64 \mathrm{MPa}$ (the maximum value of strength, which corresponds to the strength of a dry sample or quasistatically deformed water-saturated sample).

Note that the logistic function $\sigma_{U C T}(D a)$ is typical for the systems whose behavior is determined by the competition of contradirectional processes. In the considered system, such processes are compression of a solid-phase skeleton (and corresponding compression of pores) under loading and compression induced filtration of interstitial liquid. The first 
process determines the growth of pore pressure and the achievement of ultimate strength at lower values of the applied external load. The second process leads to a decrease in the local values of the pore pressure and consequently to a decrease of the contribution of this pressure to the local stress state of the skeleton (that is, to the achievement of the ultimate strength at large values of the applied external load).

Analysis of the fracture of specimens characterized by different Darcy numbers showed their similar pattern (Fig. 2). Note that with increasing Darcy number, corresponding to a decrease in the role of filtration of pore fluid and an increase in the rate of growth of pore pressure in a deformed sample, the "multiplicity" of destruction increases. As $D a$ increases, the number of main cracks and connecting secondary (local) cracks increases. In some regions, the fracture mode of concrete is actually a crushing of the cement stone material.
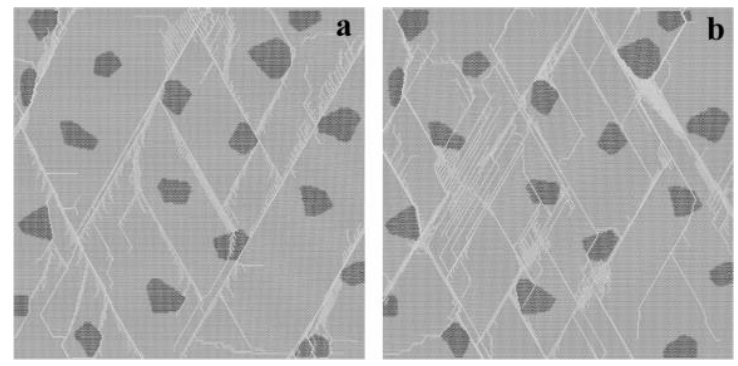

Fig. 2. Examples of fracture pattern of model water-saturated samples of concrete, characterized by different values of the analogue of the Darcy number: a) $D a=41.7$; b) $D a=10000$. Samples contain only capillary pores.

To study the contribution of micropores to the strength of samples of concretes with two-scale porosity, were also constructed the samples with two-scale porosity by adding micropores to the cement stone of the above-described samples with capillary porosity (diameter of the micropores ranged within 100-1000 microns, the microporosity was 4\%). Note that filling of micropores with liquid increases the rigidity of the sample, but the strength drops. Therefore, model tests were carried out in more "mild" conditions. Specifically, micropores were empty but capillary pores were filled with liquid. Analysis of the mechanical behavior of such samples (loading diagrams and fracture patterns) showed that micropores are the strongest stress concentrator, which reduce strength and determine the fracture pattern of the sample. The sample with micropores loses carrying capacity earlier than the excess pore pressure of the fluid in the capillaries reaches a critical value necessary for the nucleation and development of damages and cracks. Indeed, the strength of samples containing empty micropores is at least $10 \%$ lower than the minimum value of the strength of samples containing only capillary pores filled with liquid $\left(\sigma_{c}^{\min }\right)$. Therefore, the destruction of such samples is in many respects similar to the destruction of dry samples and almost does not depend of the loading rate. This result indicates that microporosity is a key factor determining strength and fracture pattern of heavy concrete. In the case of micropores filled with water, the dependence of the strength of the samples on the parameter $D a$ is similar to that shown in Fig. 1.

\section{Summary}

Based on the simulation results, it was shown that the strength of heavy water-saturated concretes is determined by the ratio of the strain rate of the solid-phase skeleton and the rate of filtration of the pore water. We found that an analogue of the Darcy number can be used as a numerical dimensionless parameter characterizing this ratio. A typical form of 
dependence of strength on a Darcy number analogue is obtained. It has a logistic profile and is approximated with good accuracy by a sigmoidal function with a power index close to 2. For small Darcy numbers (in particular, at low loading rates), the strength of concrete is determined mainly by microporosity characteristics. With an increase in the Darcy number (for example, as a result of an increase in the loading rate), the strength of the sample is determined by the parameters of the pore network of lower scale (capillary pores) as the pore liquid does not have time to redistribute into the network of capillaries. Thus, the porosity of each spatial scale makes a key contribution to the mechanical characteristics of concrete in a certain range of conditions, uniquely characterized by the corresponding range of Darcy number.

The investigation has been carried out in the framework of Presidium RAS Program 16 "Development of physical-chemical mechanics of surface phenomena as a fundamental basis for engineering design of modern structures and technologies".

\section{References}

1. I.S. Konovalenko, A.Yu. Smolin, S.G. Psakhie, Phys. Mesomech., 13(1-2), 47 (2010)

2. I.S. Konovalenko, A.Yu. Smolin, S.G. Psakhie, Frattura ed Integrita Strutturale, 24, 75 (2013)

3. I.S. Konovalenko, A.Y. Smolin, S.G. Psakhie, AIP Conf. Proc., 1683, 020089 (2015)

4. R. Balokhonov, A. Zinoviev, V. Romanova et.al, Meccanica 51(2), 415 (2016)

5. V. Romanova, R. Balokhonov, O. Emelyanova, Phys. Mesomech., 14(3-4), 159 (2011)

6. I.Yu. Smolin, P.V. Makarov, M.O. Eremin, Proc. Struct. Integr., 2, 3353 (2016)

7. S.G. Psakhie, A.V. Dimaki, E.V. Shilko et.al., Int. J. Numer. Meth. Engrg., 106, 623 (2016)

8. Varlamov A.A., Krutsilyak Yu.M, Sinitsyn A.V., European science of the 21st century: construction and architecture - 2008 (2006, URL: http://www.rusnauka.com/12_EN_2008/Stroitelstvo/31027.doc.htm).

9. S.Dutta and J.M.C.Kishen, Phys. Mesomech., 22(2), 93 (2019)

10. S. Khodaie, F. Matta, M. Alnaggar, Engineering Fracture Mechanics, 216, 106486 (2019)

11. M. A. Biot, J. Appl. Mech. 24, 594-601 (1957)

12. E. Detournay and A. H.-D. Cheng. Fundamentals of poroelasticity. in Comprehensive Rock Engineering: Principles, Practice and Projects (V.II., Ch.5, ed. Hudson, J. A., 113, Pergamon Press 1993)

13. V.N. Nikolaevsky, Geomechanics and Fluidodynamics: with Applications to Reservoir Engineering (Dordrecht, Boston: Kluwer Academic, 1996)

14. Y.P. Stefanov, M.A. Chertov, G.R. Aidagulov, A.V. Myasnikov, J Mech Phys Solids 59(11), 2323 (2011)

15. L.R. Alejano, A. Bobet, Rock Mechanics and Rock Engineering, 45(6), 995 (2012)

16. D.C. Drucker, W. Prager, Q. Appl. Math., 10(2), 157 (1952)

17. K. S. Basniev, N. M. Dmitriev, G. V. Chilingar et.al., Mechanics of Fluid Flow (John Wiley \& Sons, 2012)

18. M. Duda, J. Renner, Geophys. J. Int. 192, 1091-1108 (2013) 